\title{
Correction to: Almost periodic functions in terms of Bohr's equivalence relation
}

\author{
J. M. Sepulcre ${ }^{1} \cdot$ T. Vidal $^{1}$
}

Published online: 1 March 2019

(c) Springer Science+Business Media, LLC, part of Springer Nature 2019

\section{Correction to: Ramanujan J (2018) 46:245-267 https://doi.org/10.1007/s11139-017-9950-1}

The authors wish to draw the attention to a mistake which appears in the proof of Proposition 3 of the above quoted paper [4]. Specifically, after label (17), the statement "we can also construct a vector $\mathbf{x}_{0} \in \mathbb{R}^{\sharp G_{\Lambda}}$ such that, by taking into account Remarks 1 and 2 , verifies

$$
a_{j}=a_{1, j} e^{i\left\langle\mathbf{r}_{j}, \mathbf{x}_{0}\right\rangle}, j=1,2 \ldots, \text { with } \lambda_{j} \in \Lambda^{\prime \prime}
$$

is correct when it is possible to obtain an integral basis for the set of exponents or frequencies $\Lambda$, but it is not correct in the general case. We next define the concept of integral basis.

Let $G_{\Lambda}=\left\{g_{1}, g_{2}, \ldots, g_{k}, \ldots\right\}$ be a basis of the $\mathbb{Q}$-vector space generated by a set $\Lambda=\left\{\lambda_{1}, \lambda_{2}, \ldots\right\}$ of exponents (by abuse of notation, it is said that $G_{\Lambda}$ is a basis for $\Lambda$ ), which implies that $G_{\Lambda}$ is linearly independent over the rational numbers and each $\lambda_{j}$ is expressible as a finite linear combination of terms of $G_{\Lambda}$, say

$$
\lambda_{j}=\sum_{k=1}^{i_{j}} r_{j, k} g_{k} \quad \text { for some } r_{j, k} \in \mathbb{Q}, i_{j} \in \mathbb{N} .
$$

We will say that $G_{\Lambda}$ is an integral basis for $\Lambda$ when $r_{j, k} \in \mathbb{Z}$ for any $j, k$.

By taking into account this situation, in order to maintain the validity of Proposition 3 (not only for the case when it is possible to obtain an integral basis for the set of

The original article can be found online at https://doi.org/10.1007/s11139-017-9950-1.

\footnotetext{
J. M. Sepulcre

JM.Sepulcre@ua.es

T. Vidal

tmvg@alu.ua.es
}

1 Department of Mathematics, University of Alicante, 03080 Alicante, Spain 
exponents) and some other subsequent results of our paper, the equivalence relation which is inspired by that of Bohr is now revised to adapt correctly the situation in the general case. In this way, Definition 3 of the above quoted paper is now modified in the following terms.

Definition $3^{\prime}$ Given $\Lambda=\left\{\lambda_{1}, \lambda_{2}, \ldots, \lambda_{j}, \ldots\right\}$ a set of exponents, consider $A_{1}(p)$ and $A_{2}(p)$ two exponential sums in the class $\mathcal{S}_{\Lambda}$, say $A_{1}(p)=\sum_{j \geq 1} a_{j} e^{\lambda_{j} p}$ and $A_{2}(p)=\sum_{j \geq 1} b_{j} e^{\lambda_{j} p}$. We will say that $A_{1}$ is equivalent to $A_{2}$ if for each integer value $n \geq 1$, with $n \leq \sharp \Lambda$, it is satisfied $a_{n}^{*} \sim b_{n}^{*}$, where $a_{n}^{*}, b_{n}^{*}:\left\{\lambda_{1}, \lambda_{2}, \ldots, \lambda_{n}\right\} \rightarrow \mathbb{C}$ are the functions given by $a_{n}^{*}\left(\lambda_{j}\right):=a_{j}$ y $b_{n}^{*}\left(\lambda_{j}\right):=b_{j}, j=1,2, \ldots, n$ and $\sim$ is in Definition 1.

It is clear that the relation defined in the foregoing definition is an equivalence relation. That is, it is reflective, symmetric and transitive. By abuse of notation, we will use $\sim$ for both equivalence relations introduced in Definitions 1 and $3^{\prime}$.

Analogously, Definition 5 must be rewritten in the following terms.

Definition $5^{\prime}$ Given $\Lambda=\left\{\lambda_{1}, \lambda_{2}, \ldots, \lambda_{j}, \ldots\right\}$ a set of exponents, let $f_{1}$ and $f_{2}$ denote two equivalence classes of $B(\mathbb{R}, \mathbb{C}) / \simeq(\operatorname{resp} . B(U, \mathbb{C}) / \simeq)$ whose associated Fourier series (resp. Dirichlet series) are given by

$$
\begin{aligned}
& \sum_{j \geq 1} a_{j} e^{i \lambda_{j} t} \text { and } \sum_{j \geq 1} b_{j} e^{i \lambda_{j} t}, a_{j}, b_{j} \in \mathbb{C}, \lambda_{j} \in \Lambda . \\
& \left(\operatorname{resp} . \sum_{j \geq 1} a_{j} e^{\lambda_{j} s} \text { and } \sum_{j \geq 1} b_{j} e^{\lambda_{j} s}, a_{j}, b_{j} \in \mathbb{C}, \lambda_{j} \in \Lambda .\right)
\end{aligned}
$$

We will say that $f_{1}$ is equivalent to $f_{2}$ if for each integer value $n \geq 1$, with $n \leq \sharp \Lambda$, it is satisfied $a_{n}^{*} \sim b_{n}^{*}$, where $a_{n}^{*}, b_{n}^{*}:\left\{\lambda_{1}, \ldots, \lambda_{n}\right\} \rightarrow \mathbb{C}$ are the functions given by $a_{n}^{*}\left(\lambda_{j}\right):=a_{j}$ and $b_{n}^{*}\left(\lambda_{j}\right):=b_{j}, j=1,2, \ldots, n$, and $\sim$ is in Definition 1.

Under the modification of Definition 3, Proposition 1 must now be rewritten in the following way.

Proposition $1^{\prime}$ Given $\Lambda=\left\{\lambda_{1}, \lambda_{2}, \ldots, \lambda_{j}, \ldots\right\}$ a set of exponents, consider $A_{1}(p)$ and $A_{2}(p)$ two exponential sums in the class $\mathcal{S}_{\Lambda}$, say $A_{1}(p)=\sum_{j \geq 1} a_{j} e^{\lambda_{j} p}$ and $A_{2}(p)=\sum_{j \geq 1} b_{j} e^{\lambda_{j} p}$. Fixed a basis $G_{\Lambda}$ for $\Lambda$, for each $j \geq 1$ let $\mathbf{r}_{j} \in \mathbb{R}^{\sharp G_{\Lambda}}$ be the vector of rational components verifying (2). Then $A_{1} \sim A_{2}$ if and only if for any integer value $n \geq 1$, with $n \leq \sharp \Lambda$, there exists $\mathbf{x}_{n}=\left(x_{n, 1}, x_{n, 2}, \ldots, x_{n, k}, \ldots\right) \in \mathbb{R}^{\sharp G_{\Lambda}}$ such that $b_{j}=a_{j} e^{\left\langle\mathbf{r}_{j}, \mathbf{x}_{n}\right\rangle i}$ for $j=1,2, \ldots, n$. Furthermore, if $G_{\Lambda}$ is an integral basis for $\Lambda$ then $A_{1} \sim A_{2}$ if and only if there exists $\mathbf{x}_{0}=\left(x_{0,1}, x_{0,2}, \ldots, x_{0, k}, \ldots\right) \in \mathbb{R}^{\sharp G_{\Lambda}}$ such that $b_{j}=a_{j} e^{\left\langle\mathbf{r}_{j}, \mathbf{x}_{0}\right\rangle i}$ for every $j \geq 1$.

Proof For each integer value $n \geq 1$, let $V_{n}$ be the $\mathbb{Q}$-vector space generated by $\left\{\lambda_{1}, \ldots, \lambda_{n}\right\}, V$ the $\mathbb{Q}$-vector space generated by $\Lambda$, and $G_{\Lambda}=\left\{g_{1}, g_{2}, \ldots, g_{k}, \ldots\right\}$ a basis of $V$. If $A_{1} \sim A_{2}$, by Definition $3^{\prime}$ for any integer value $n \geq 1$, with $n \leq \sharp \Lambda$, there exists a $\mathbb{Q}$-linear map $\psi_{n}: V_{n} \rightarrow \mathbb{R}$ such that

$$
b_{j}=a_{j} e^{i \psi_{n}\left(\lambda_{j}\right)}, j=1,2 \ldots, n .
$$


Hence $b_{j}=a_{j} e^{i \sum_{k=1}^{i_{j}} r_{j, k} \psi_{n}\left(g_{k}\right)}, \quad j=1,2 \ldots, n$ or, equivalently,

$$
b_{j}=a_{j} e^{i\left\langle\mathbf{r}_{j}, \mathbf{x}_{n}\right\rangle}, \quad j=1,2 \ldots, n,
$$

with $\mathbf{x}_{n}:=\left(\psi_{n}\left(g_{1}\right), \psi_{n}\left(g_{2}\right), \ldots\right)$. Conversely, suppose the existence, for any integer value $n \geq 1$ with $n \leq \sharp \Lambda$, of a vector $\mathbf{x}_{n}=\left(x_{n, 1}, x_{n, 2}, \ldots, x_{n, k}, \ldots\right) \in \mathbb{R}^{\sharp G_{\Lambda}}$ such that $b_{j}=a_{j} e^{\left\langle\mathbf{r}_{j}, \mathbf{x}_{n}\right\rangle \hat{i}}, j=1,2 \ldots, n$. Thus a $\mathbb{Q}$-linear map $\psi_{n}: V_{n} \rightarrow \mathbb{R}$ can be defined from $\psi_{n}\left(g_{k}\right):=x_{n, k}, k \geq 1$. Therefore

$$
\psi_{n}\left(\lambda_{j}\right)=\sum_{k=1}^{i_{j}} r_{j, k} \psi\left(g_{k}\right)=\left\langle\mathbf{r}_{j}, \mathbf{x}_{n}\right\rangle, j=1,2 \ldots, n,
$$

and the result follows.

Now, suppose that $G_{\Lambda}$ is an integral basis for $\Lambda$ and $A_{1} \sim A_{2}$. Thus, by above, for each fixed integer value $n \geq 1$, let $\mathbf{x}_{n}=\left(x_{n, 1}, x_{n, 2}, \ldots\right) \in \mathbb{R}^{\sharp G_{\Lambda}}$ be a vector such that

$$
b_{j}=a_{j} e^{i\left\langle\mathbf{r}_{j}, \mathbf{x}_{n}\right\rangle}, j=1,2 \ldots, n \text {. }
$$

Since each component of $\mathbf{r}_{j}$ is an integer number, without loss of generality, we can take $\mathbf{x}_{n} \in[0,2 \pi)^{\sharp G_{\Lambda}}$ as the unique vector in $[0,2 \pi)^{\sharp G_{\Lambda}}$ satisfying the above equalities, where we assume $x_{n, k}=0$ for any $k$ such that $r_{j, k}=0$ for $j=1, \ldots, n$. Therefore, under this assumption, if $m>n$ then $x_{m, k}=x_{n, k}$ for any $k$ so that $x_{n, k} \neq 0$. In this way, we can construct a vector $\mathbf{x}_{0}=\left(x_{0,1}, x_{0,2}, \ldots, x_{0, k}, \ldots\right) \in[0,2 \pi)^{\sharp G_{\Lambda}}$ such that $b_{j}=a_{j} e^{\left\langle\mathbf{r}_{j}, \mathbf{x}_{0}\right\rangle i}$ for every $j \geq 1$. Indeed, if $r_{1, k} \neq 0$ then the component $x_{0, k}$ is chosen as $x_{1, k}$, and if $r_{1, k}=0$ then each component $x_{0, k}$ is defined as $x_{n+1, k}$ where $r_{j, k}=0$ for $j=1, \ldots, n$ and $r_{n+1, k} \neq 0$. Conversely, if there exists $\mathbf{x}_{0}=$ $\left(x_{0,1}, x_{0,2}, \ldots, x_{0, k}, \ldots\right) \in \mathbb{R}^{\sharp G_{\Lambda}}$ such that $b_{j}=a_{j} e^{\left\langle\mathbf{r}_{j}, \mathbf{x}_{0}\right\rangle i}$ for every $j \geq 1$, then it is clear that $A_{1} \sim A_{2}$ under Definition $3^{\prime}$.

From Proposition $1^{\prime}$, it is worth noting that old Definition 3 and Definition $3^{\prime}$ are equivalent in the case that it is possible to obtain an integral basis for the set of exponents $\Lambda$. Consequently, all the results of the quoted above paper which can me formulated in terms of an integral basis are also valid under old Definition $3^{\prime}$ (in particular, the mistake above does not concern the results on the finite exponential sums in Section 3 and the Riemann zeta function in Section 5). Moreover, in this case, the set of all exponential sums $A(p)$ in an equivalence class $\mathcal{G}$ in $\mathcal{S}_{\Lambda} / \sim$ can be determined by a function $E_{\mathcal{G}}: \mathbb{R}^{\sharp G_{\Lambda}} \rightarrow \mathcal{S}_{\Lambda}$ of the form

$$
E_{\mathcal{G}}(\mathbf{x}):=\sum_{j \geq 1} a_{j} e^{\left\langle\mathbf{r}_{j}, \mathbf{x}\right\rangle i} e^{\lambda_{j} p}, \mathbf{x}=\left(x_{1}, x_{2}, \ldots, x_{k}, \ldots\right) \in \mathbb{R}^{\sharp G_{\Lambda}},
$$

where $a_{1}, a_{2}, \ldots, a_{j}, \ldots$ are the coefficients of an exponential sum in $\mathcal{G}$ and the $\mathbf{r}_{j}$ 's are the vectors associated with a prefixed integral basis $G_{\Lambda}$ for $\Lambda$. 
However, both Definitions 3 and $3^{\prime}$ are not equivalent in the general case. For example, consider the set of exponents $\Lambda_{0}=\left\{\lambda_{1}, \lambda_{2}, \ldots, \lambda_{j}, \ldots\right\}$ given by

$$
\lambda_{j}=2 j-1+\frac{1}{2(2 j-1)} \in \mathbb{Q}, j=1,2, \ldots,
$$

then $G_{\Lambda_{0}}=\{1\}$ is a basis for $\Lambda_{0}$ but it is not an integral basis for $\Lambda_{0}$. In fact, it is not possible to get an integral basis for $\Lambda_{0}$. Now, consider the exponential sums $B_{1}(p)=$ $\sum_{j \geq 1} e^{-\lambda_{j} p}$ and $B_{2}(p)=-B_{1}(p)=\sum_{j \geq 1}-e^{-\lambda_{j} p}$. Then $B_{1}(p)$ and $B_{2}(p)$ are equivalent according to Definition $3^{\prime}$. Indeed, take $a_{j}=1$ and $b_{j}=-1$ for each $j=1,2, \ldots, G_{\Lambda_{0}}=\{1\}, r_{j}=\lambda_{j}$ for each $j=1,2, \ldots$ and $x_{n}=2 \pi \prod_{k=1}^{n}(2 k-1)$, $n=1,2, \ldots$. Fixed $n \geq 1$, then it is clear that $b_{j}=a_{j} e^{\left\langle r_{j}, x_{n}\right\rangle i}$ for every $j=$ $1,2, \ldots, n$, i.e.

$$
-1=e^{i\left(2 j-1+\frac{1}{2(2 j-1)}\right) 2 \pi \prod_{k=1}^{n}(2 k-1)}, \quad j=1,2, \ldots, n .
$$

Nevertheless, as it is deduced from Bohr's paper [2], $B_{1}(p)$ and $B_{2}(p)$ are not equivalent according to old Definition 3.

Now, mutatis mutandi, the proofs of Lemma 1, Lemma 2 and Proposition 3 can be rewritten. Specifically, the modifications are as follows.

- In the proof of Lemma 1: "taking $\mathbf{x}_{0}=\tau \mathbf{g}$ " must be changed by "taking $\mathbf{x}_{n}=\tau \mathbf{g}$ for any integer value $n \geq 1$ ".

- In the proof of Lemma 2: "there exists $\mathbf{x}_{0} \in \mathbb{R}^{\sharp \Lambda}$ such that $b_{j}=a_{j} e^{\left\langle\mathbf{r}_{j}, \mathbf{x}_{0}\right\rangle i}$ for each $j \geq 1$ " must be changed by "for any integer value $n \geq 1$, with $n \leq \sharp \Lambda$, there exists $\mathbf{x}_{n} \in \mathbb{R}^{\sharp \Lambda}$ such that $b_{j}=a_{j} e^{\left\langle\mathbf{r}_{j}, \mathbf{x}_{n}\right\rangle i}$ for each $j=1, \ldots, n$ ".

- In the proof of Lemma 2: "define the sequence of trigonometric polynomials

$$
Q_{k}(t):=\sum_{j \geq 1} p_{j, k} a_{j} e^{\left\langle\mathbf{r}_{j}, \mathbf{x}_{0}\right\rangle i} e^{i \lambda_{j} t}, \quad k=1,2, \ldots ”
$$

must be changed by "define the sequence of trigonometric polynomials

$$
Q_{k}(t):=\sum_{j \geq 1} p_{j, k} b_{j} e^{i \lambda_{j} t}, \quad k=1,2, \ldots,
$$

where, fixed $k$, we can take $b_{j}=a_{j} e^{\left\langle\mathbf{r}_{j}, \mathbf{x}_{n_{k}}\right\rangle i}$ with $n_{k}$ the greatest integer value $j$ such that $p_{j, k} \neq 0 . "$

- In the proof of Lemma 2: "However, note that

$$
\begin{aligned}
M\left\{\left|Q_{k_{1}}(t)-Q_{k_{2}}(t)\right|^{2}\right\} & =\sum_{j \geq 1}\left(p_{j, k_{1}}-p_{j, k_{2}}\right)^{2}\left|e^{\left\langle\mathbf{r}_{j}, \mathbf{x}_{0}\right\rangle i}\right|^{2}\left|a_{j}\right|^{2} \\
& \left.=M\left\{\left|P_{k_{1}}(t)-P_{k_{2}}(t)\right|^{2}\right\}\right\}, "
\end{aligned}
$$


must be changed by "However, note that

$$
\begin{aligned}
M\left\{\left|Q_{k_{1}}(t)-Q_{k_{2}}(t)\right|^{2}\right\} & =\sum_{j \geq 1}\left(p_{j, k_{1}}-p_{j, k_{2}}\right)^{2}\left|e^{\left\langle\mathbf{r}_{j}, \mathbf{x}_{n_{2}}\right\rangle i}\right|^{2}\left|a_{j}\right|^{2} \\
& =M\left\{\left|P_{k_{1}}(t)-P_{k_{2}}(t)\right|^{2}\right\}
\end{aligned}
$$

where we suppose that $n_{k_{2}} \geq n_{k_{1}}$."

- In the proof of Lemma 2: "we have $f_{2}(t) \in A P(\mathbb{R}, \mathbb{C})$. Moreover, $\left\{Q_{k}(t)\right\}_{k \geq 1}$ also converges formally to the series $\sum_{j \geq 1} a_{j} e^{\left\langle\mathbf{r}_{j}, \mathbf{x}_{0}\right\rangle i} e^{i \lambda_{j} t}$, which, by [1, p. 21], represents the Fourier series of $f_{2}(t)$. Finally, by taking into account Definition 5 (in terms of Proposition 1) we have $f_{1} \sim f_{2}$." must be changed by "we have $f_{2}(t) \in A P(\mathbb{R}, \mathbb{C})$ and, by $\left[1\right.$, p. 21], $\sum_{j \geq 1} b_{j} e^{i \lambda_{j} t}$ represents its Fourier series. Finally, by taking into account Definition 5 (in terms of Proposition $1^{\prime}$ ) we have $f_{1} \sim f_{2}$."

- In the proof of Proposition 3: "Since $f_{1} \sim f_{l}$ for each $l=1,2, \ldots$, we deduce from Proposition 1 that there exists $\mathbf{x}_{l}=\left(x_{l, 1}, x_{l, 2}, \ldots\right) \in \mathbb{R}^{\sharp G_{\Lambda}}$ such that

$$
a_{l, j}=a_{1, j} e^{i\left\langle\mathbf{r}_{j}, \mathbf{x}_{l}\right\rangle}, j=1,2 \ldots, \text { with } \lambda_{j} \in \Lambda . "
$$

must be changed by "Since $f_{1} \sim f_{l}$ for each $l=1,2, \ldots$, we deduce from Proposition $1^{\prime}$ that for any integer value $n \geq 1$, with $n \leq \sharp \Lambda$, there exists $\mathbf{x}_{l, n}=$ $\left(x_{l, n, 1}, x_{l, n, 2}, \ldots\right) \in \mathbb{R}^{\sharp G_{\Lambda}}$ such that

$$
a_{l, j}=a_{1, j} e^{i\left\langle\mathbf{r}_{j}, \mathbf{x}_{l, n}\right\rangle}, j=1,2 \ldots, n \text { with } \lambda_{j} \in \Lambda . "
$$

- In the proof of Proposition 3: "and, since (16) is satisfied for any $k=1,2, \ldots$, we can also construct a vector $\mathbf{x}_{0} \in \mathbb{R}^{\sharp G_{\Lambda}}$ such that, by taking into account remarks 1 and 2 , verifies

$$
a_{j}=a_{1, j} e^{i\left\langle\mathbf{r}_{j}, \mathbf{x}_{0}\right\rangle}, j=1,2 \ldots, \text { with } \lambda_{j} \in \Lambda . "
$$

must be changed by "and, since (16) is satisfied for any $k=1,2, \ldots$, we can construct, for any integer value $n \geq 1$ with $n \leq \sharp \Lambda$, a vector $\mathbf{x}_{0, n} \in \mathbb{R}^{\sharp G_{\Lambda}}$ such that, by taking into account remarks 1 and 2, verifies

$$
a_{j}=a_{1, j} e^{i\left\langle\mathbf{r}_{j}, \mathbf{x}_{0, n}\right\rangle}, j=1,2 \ldots, n \text { with } \lambda_{j} \in \Lambda . "
$$

The rest of the article remains unchanged.

Finally, the authors wish to thank Mattia Righetti for his quote to Bohr's example given by the set of exponents $\Lambda_{0}$ considered above [2,3].

\section{References}

1. Besicovitch, A.S.: Almost Periodic Functions. Dover, New York (1954) 
2. Bohr, H.: Zur Theorie der allgemeinen Dirichletschen Reihen. Math. Ann. (German) 79, 136-156 (1918)

3. Righetti, M.: On Bohr's equivalence theorem. J. Math. Anal. Appl. (Corrigendum Ibid.) 449, 939-940 (2017)

4. Sepulcre, J.M., Vidal, T.: Almost periodic functions in terms of Bohr's equivalence relation. Ramanujan J. 46(1), 245-267 (2018)

Publisher's Note Springer Nature remains neutral with regard to jurisdictional claims in published maps and institutional affiliations. 